\title{
A characterization of the multivariate excess wealth ordering
}

\author{
Author's version \\ Published in Insurance: Mathematics and Economics 49 (2011) 410417 \\ doi:10.1016/j.insmatheco.2011.07.001 \\ http://www.sciencedirect.com/science/article/pii/S0167668711000783
}

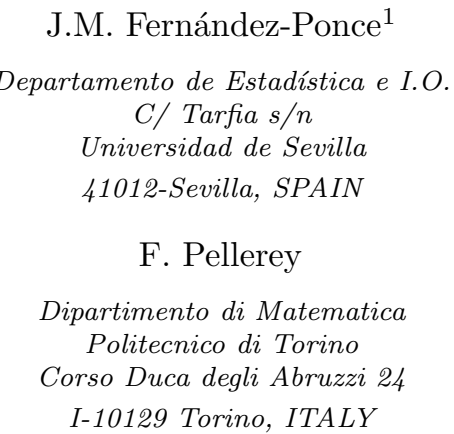

Rodríguez-Griñolo, M.R.

Departamento Economía, Métodos Cuantitativos e Historia Económica Universidad Pablo de Olavide

Ctra. de Utrera, Km 1

41013 Sevilla, SPAIN.

\begin{abstract}
In this paper, some new properties of the upper-corrected orthant of a random vector are proved. The univariate rightspread or excess wealth function, introduced by Fernández-Ponce et al. (1996), is extended to multivariate random vectors, and some properties of this multivariate function are studied. Later, this function was used to define the excess wealth ordering by Shaked and Shanthikumar (1998) and Fernández-Ponce et al. (1998). The multivariate excess wealth function enable us to define a new stochastic comparison which is weaker than the multivariate dispersion orderings. Also, some properties relating the multivariate excess wealth order with stochastic dependence are described.
\end{abstract}

Keywords: Excess Wealth Function; Expansion Function; Multivariate Dispersion Ordering; Quantile; Upper-corrected Orthant.

\section{Introduction}

Comparisons among univariate random variables in some stochastic sense have been extensively studied by many authors during the last thirty years. There exist many applications of these stochastic orderings, from economic theory to reliability and queueing theory (see Barlow and Proshan, 1975; Stoyan, 1983; Shaked and Shanthikumar, 1994). In particular, variability orders for univariate distributions have found a profound interest among researchers. Among these types of orders, the dispersion ordering has

\footnotetext{
${ }^{1}$ Corresponding author
}

been well studied. Two interesting papers on the dispersion ordering (d.o.) are the ones by Shaked (1982) and Deshpandé and Kochar (1983). This order was characterized through the number of crossings and the corresponding changes of sign for the distribution functions (see Hickey, 1986; Muñóz-Pérez, 1990; Saunders, 1984, and Bartoszewicz, 1995). Bartoszewicz (1995) characterized the d.o. using the TTT transforms and he found a relationship between the order based on the mean residual lives and the d.o. (see Bartoszewicz, 1997). Kochar (1996) studied the d.o. among order statistics from DFR distributions.

The d.o. has been also considered to characterize lifetime 
distributions satisfying particular aging properties; different characterizations for IFR and DFR random variables by means of the d.o can be seen, for example, in Pellerey and Shaked (1997). Other types of aging properties for lifetime distributions, weaker than IFR, have been proposed in Kochar and Wiens (1987). Thus, a characterization in dispersion sense for these distributions needed of a new comparison again based on dispersion but weaker than the classic d.o. For this reason, Fernández-Ponce et al. (1996) gave the concept of right-spread function, which characterized the aging notions defined in in Kochar and Wiens (1987). In a parallel direction and in an independent way, Shaked and Shanthikumar (1998) defined the same function which was named by them as the excess wealth function. The analysis of a new weak dispersion ordering, which was called excess wealth order, was developed in Shaked and Shanthikumar (1998) and FernándezPonce et al. (1998). Later several authors have characterized lifetime distributions using this partial order (see Kochar and Carrière, 1997 and Kochar, et al., 2001 among others).

In this paper, a multivariate generalization of the excess wealth function is defined and studied, and a multivariate stochastic comparison is introduced. The relationships between this comparison and other orders based on dispersion, and in dependence properties, are investigated. Also, a new characterization of the CIS (Conditionally Increasing in Sequence) property, whose definition is recalled next, is provided.

The paper is organized in the following way. In Section 2, we further study some properties of the multivariate quantiles which were introduced in Fernández-Ponce and Suárez-Lloréns (2003). In Section 3 , we extend the concept of excess wealth function to the multivariate case. In Section 4, the excess wealth order for multivariate distributions is defined and studied. For example, it is shown that the multivariate excess-wealth order is weaker than the multivariate dispersion ordering.

\section{Notation and preliminaries}

Some notation which will be used throughout the paper is given here. The usual stochastic ordering between univariate random variables is denoted by $\leq_{S T}$, that is, $X \leq_{S T} Y$ if $P[X \leq x] \geq P[Y \leq x]$ for all $x$ in $\mathbb{R}$. Fundamentally, random vectors will be dealt with which take on values in $\mathbb{R}^{n}$. The space $\mathbb{R}^{n}$ is endowed with the usual componentwise partial order, which is defined as follows: let $\mathbf{x}=\left(x_{1}, \cdots, x_{n}\right)$ and $\mathbf{y}=\left(y_{1}, \cdots, y_{n}\right)$ be two vectors in $\mathbb{R}^{n}$, then $\mathbf{x} \leq \mathbf{y}$ if $x_{i} \leq y_{i}$ for $i=1, \cdots, n$. Throughout the paper "increasing" means "non-decreasing" and "decreasing" means "non-increasing". Particularly, a function $\phi: \mathbb{R}^{n} \longrightarrow \mathbb{R}^{n}$ is said to be an increasing function when $\phi(\mathbf{x}) \leq \phi(\mathbf{y})$ for $\mathbf{x} \leq \mathbf{y}$. The notation $\sim_{s t}$ stands for equality in law. The vector of ones will be denoted by $\mathbf{1}$, i.e. $\mathbf{1}=(1, \cdots, 1)$, and the corresponding of zeros by $\mathbf{0}$. The multiple integral $\int_{A} F\left(t_{1}, \ldots, t_{n}\right) d t_{1} \ldots d t_{n}$ will be denoted as $\int_{A} F(\mathbf{t}) d \mathbf{t}$. The dimension of a random vector is clear from the context and unless otherwise stated it is assumed that it is $n$.

Let $\mathbf{X}$ be a random vector in $\mathbb{R}^{n}$ with distribution function (cdf) $F(\cdot)$. The multivariate $\mathbf{u}$-quantile for $\mathbf{X}$, also called regression representation, was introduced by $\mathrm{O}$ 'Brien (1975), Arjas and Lehtonen (1978) and Rüschendorf (1981). The definition is as follows. Let $\mathbf{u}_{n}=\left(u_{1}, \ldots, u_{n}\right)$ be a vector in $[0,1]^{n}$, the multivariate $\mathbf{u}$-quantile for $\mathbf{X}$, denoted by $\hat{\mathbf{x}}\left(\mathbf{u}_{n}\right)$, is defined as

$$
\begin{array}{r}
\hat{x}_{1}\left(u_{1}\right)=F_{X_{1}}^{-}\left(u_{1}\right), \hat{x}_{2}\left(\mathbf{u}_{2}\right)=F_{X_{2} \mid X_{1}=\hat{x}_{1}\left(u_{1}\right)}^{-}\left(u_{2}\right), \ldots, \\
\hat{x}_{n}\left(\mathbf{u}_{n}\right)=F_{X_{n} \mid \bigcap_{j=1}^{n-1} X_{j}=\hat{x}_{j}\left(\mathbf{u}_{j}\right)}^{-}\left(u_{n}\right)
\end{array}
$$

where $F^{-}(u)=\inf \{x: F(x) \geq u\}$ and $\mathbf{u}_{i}=$ $\left(u_{1}, \ldots, u_{i}\right)$ for all $i=1, \ldots, n$.

Müller and Scarsini (2001) used the multivariate uquantile to provide conditions for the stochastic comparison of random vectors with a common copula. $\mathrm{Li}$ et al. (1996) used this representation as a tool for the construction of multivariate distributions with given nonoverlapping multivariate marginals. Shaked and Shanthikumar (1998) also proposed the standard construction as an useful tool for the stochastic comparison of random vectors. Rüschendorf (2004) used it for the stochastic comparison of risks with respect to supermodular ordering which is of particular interest in many applications.

More recently, Fernández-Ponce and Suárez-Lloréns (2003) also defined several concepts for a multivariate random vector which will be used later on. The first concept is the multivariate $\mathbf{x}$-rate vector, denoted by $\stackrel{\star}{\mathbf{x}}(\mathbf{x})$, and it is defined as $\stackrel{\star}{x}_{1}\left(x_{1}\right)=P\left(X_{1} \leq x_{1}\right), \stackrel{\star}{x}_{2}\left(\mathbf{x}_{2}\right)=P\left(X_{2} \leq\right.$ $\left.x_{2} \mid X_{1}=x_{1}\right), \ldots . . \stackrel{\star}{x}_{n}\left(\mathbf{x}_{n}\right)=P\left(X_{n} \leq x_{n} \mid \bigcap_{j=1}^{n-1} X_{j}=x_{j}\right)$, where $\mathbf{x}_{i}=\left(x_{1}, \ldots, x_{i}\right)$ for all $i=1, \ldots, n$. The second concept is the right-upper orthant at a point $\mathbf{z}$, denoted by $C(\mathbf{z})$, and it is defined as $C(\mathbf{z})=\left\{\mathbf{x} \in \mathbb{R}^{n}: \mathbf{z} \leq \mathbf{x}\right\}$. At the end, the upper-corrected orthant at a point $\mathbf{z}$ for the random variable $\mathbf{X}$, denoted as $R_{\mathbf{X}}(\mathbf{z})$, is defined as

$$
\begin{array}{r}
R_{\mathbf{X}}(\mathbf{z})=\left\{\mathbf{x} \in \mathbb{R}^{n}: x_{1} \geq F_{X_{1}}^{-}\left(\stackrel{(}{x}_{1}\left(z_{1}\right)\right), \ldots,\right. \\
\left.x_{n} \geq F_{X_{n} \mid \cap_{j=1}^{n-1} X_{j}=x_{j}}^{-}\left(\stackrel{\star}{x}_{n}\left(\mathbf{z}_{n}\right)\right)\right\} .
\end{array}
$$

For notational purposes, to reduce the complexity in the proofs of the results, a slight modification of this notion is considered here, restricting the set only to the points that are in the support of the vector. By taking into account that the support of a random vector $\mathbf{X}$ is defined as $\operatorname{Supp}(\mathbf{X})=\left\{\mathbf{x} \in \mathbb{R}^{n}: \mathrm{P}\left[\mathbf{X} \in B_{\mathbf{x}}(\varepsilon)\right]>0\right.$ for all $\left.\varepsilon>0\right\}$ where $B_{\mathbf{x}}(\varepsilon)$ is the centered ball at $\mathbf{x}$ with radius $\varepsilon$, then the following definition can be given. 
Definition 1. Let $\mathbf{X}$ be a random vector. Its upper corrected orthant at $\mathbf{z} \in \operatorname{Supp}(\mathbf{X})$ is defined as

$$
\begin{gathered}
R_{\mathbf{X}}(\mathbf{z})=\left\{\mathbf{x} \in \operatorname{Supp}(\mathbf{X}): x_{1} \geq F_{X_{1}}^{-}\left(\stackrel{\star}{x}_{1}\left(z_{1}\right)\right), \ldots,\right. \\
\left.x_{n} \geq F_{X_{n} \mid \cap_{j=1}^{n-1} X_{j}=x_{j}}^{-}\left({ }^{\star} x_{n}\left(\mathbf{z}_{n}\right)\right)\right\} .
\end{gathered}
$$

It is easily shown that if $\mathbf{X}$ is a random vector with independent components then $R_{\mathbf{X}}(\mathbf{z})=C(\mathbf{z}) \cap \operatorname{Supp}(\mathbf{X})$.

Remark 1. Note that if $\mathbf{t}_{1} \leq \mathbf{t}_{2}$ then it could not be held that $R_{\mathbf{X}}\left(\mathbf{t}_{2}\right) \subset R_{\mathbf{X}}\left(\mathbf{t}_{1}\right)$. For example, let $\mathbf{X}$ be a bivariate random vector with joint density function given by

$$
f_{\mathbf{X}}(\mathbf{t})= \begin{cases}2 / 3 & \text { if } \mathbf{t} \in T_{1} \\ 4 / 3 & \text { if } \mathbf{t} \in T_{2} \\ 0 & \text { otherwise }\end{cases}
$$

where $T_{1}$ is the triangle with vertices $(0,0),(0,1)$ and $(1,1)$, and $T_{2}$ is the triangle with vertices $(0,0),(1,0)$ and $(1,1)$.

Let $\mathbf{t}_{1}=\left(\frac{1}{2}, \frac{1}{2}\right)$. By straightforward computations, it is verified that

$$
R_{\mathbf{X}}\left(\mathbf{t}_{1}\right)=\left\{x \geq \frac{1}{2}, y \geq \frac{x+1}{3}\right\} .
$$

Now, consider $\mathbf{t}_{2}=\left(\frac{2}{3}, \frac{1}{2}\right)$. It holds that $\mathbf{t}_{1} \leq \mathbf{t}_{2}$, and $\mathbf{t}_{2} \in R_{\mathbf{X}}\left(\mathbf{t}_{2}\right)$. But, $\mathbf{t}_{2} \notin R_{\mathbf{X}}\left(\mathbf{t}_{1}\right)$. Thus $R_{\mathbf{X}}\left(\mathbf{t}_{2}\right) \nsubseteq R_{\mathbf{X}}\left(\mathbf{t}_{1}\right)$.

From now on, assume that the following regularity conditions $(R C)$ are verified by every $\operatorname{cdf} F$ in the paper.

1. $F$ is a continuous function.

2. The vector $\widehat{\mathbf{x}}(\mathbf{u})$ is differentiable at each component.

3. The conditional distribution of $X_{i}$ to $X_{1}, \ldots, X_{i-1}$ $\left(F_{i \mid 1, \ldots, i-1}\right)$ is a continuous and strictly increasing function for $i=1, \ldots, n$. For convenience $F_{1 \mid 0}=F_{1}$.

4. $F_{X_{i} \mid X_{1}, \ldots, X_{i-1}}^{-}(0)<\infty$ for all $i=1, \ldots, n$.

It is easy to verify that under the regularity conditions above there is a one to one correspondence between vectors $\mathbf{u}_{n} \in[0,1)^{n}$ and the points $\mathbf{x} \in \operatorname{Supp}(\mathbf{X})$.

The next result is the main reason of interest in the notion of upper corrected orthant. Its proof directly follows from Proposition 2.1 in Fernández-Ponce and Suárez-Lloréns (2003).

Proposition 1. Let $\mathbf{X}$ be a random vector. Then

$$
\mathrm{P}\left[\mathbf{X} \in R_{\mathbf{X}}\left(\widehat{\mathbf{x}}\left(\mathbf{u}_{n}\right)\right)\right]=\prod_{j=1}^{n}\left(1-u_{j}\right)
$$

for all $\mathbf{u}_{n} \in[0,1)^{n}$.

Furthermore, let $\mathbf{t}=\left(t_{1}, t_{2}\right)$ be a point in $\operatorname{Supp}(\mathbf{X})$. Then it is easily shown that

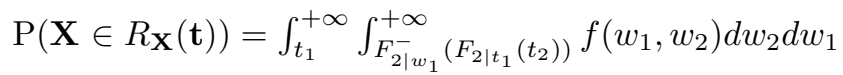
$=\quad \int_{t_{1}}^{+\infty} f_{X_{1}}\left(w_{1}\right) \int_{F_{2 \mid w_{1}}^{-}}^{+\infty}\left(F_{2 \mid t_{1}}\left(t_{2}\right)\right) f_{X_{2} \mid X_{1}=w_{1}}\left(w_{2}\right) d w_{2} d w_{1}$. Consequently, it is obtained that

$$
P\left(\mathbf{X} \in R_{\mathbf{X}}(\mathbf{t})\right)=P\left(X_{1}>t_{1}\right) P\left(X_{2}>t_{2} \mid X_{1}=t_{1}\right) .
$$

This equality can easily be generalized for the $n$-dimensional case by using induction arguments.

This result means that the probability associated to the upper corrected orthant at the $\mathbf{u}_{n}$-quantile does not depend on the distribution function.

As it can be seen in Proposition 1, the upper corrected othant plays in the multivariate setting the same role as the $u$-quantile upper orthant for univariate distributions, being $\mathrm{P}\left[X \in R_{X}(\widehat{x}(u))\right]=\mathrm{P}\left[X \geq F_{X}^{-}(u)\right]=1-u$ in the univariate case. Thus, it is interesting to describe the main properties of upper corrected orthants.

It is well-known that the univariate quantile function is an increasing function, i.e. $u \leq v$ if, and only if $Q_{X}(u) \leq$ $Q_{X}(v)$. This property is not verified by random vectors, in general. However if a type of dependence is held then this property can be verified. This type of dependence must be based on the growth of the corresponding conditional distributions. In this way, the following definition of conditionally increasing in sequence property can be found in Barlow and Proschan (1975). Also, two interesting results which will be used later are proved.

Definition 2. The random variables $X_{1}, \ldots, X_{n}$ are conditionally increasing [decreasing] in sequence (CIS) [CDS] if $X_{i}$ is stochastically increasing [decreasing] in $X_{1}, \ldots, X_{i-1}$ for $i=2, \ldots, n$.

Theorem 1. $\mathbf{X}$ is a CIS random vector if and only if $R_{\mathbf{X}}(\mathbf{x}) \subset C(\mathbf{x})$ for all $\mathbf{x} \in \mathbb{R}^{n}$.

Proof. First, we prove the sufficient condition. The proof will be by mathematical induction. The proposition is obviously true for $n=1$. Assume that the proposition is true for $n=m$. We now need to show that it is true for $n=m+1$. If $\widehat{\mathbf{x}}\left(\mathbf{u}_{m}\right) \leq \mathbf{t}_{m}$ then, given that $\mathbf{X}$ is CIS,

$$
\widehat{x}_{m+1 \mid \widehat{\mathbf{x}}_{m}\left(\mathbf{u}_{m}\right)}\left(u_{m+1}\right) \leq \widehat{x}_{m+1 \mid \mathbf{t}_{m}}\left(u_{m+1}\right) \text {. }
$$

However, we know that if $\mathbf{t}_{m+1} \in R_{\mathbf{X}}\left[\widehat{\mathbf{x}}\left(\mathbf{u}_{m+1}\right)\right]$ then $t_{m+1} \geq \widehat{x}_{m+1 \mid \mathbf{t}_{m}}\left(u_{m+1}\right)$. Therefore, $\mathbf{t}_{m+1} \in C\left[\widehat{\mathbf{x}}\left(\mathbf{u}_{m+1}\right)\right]$. Hence we have completed the proof by the induction argument.

The necessary condition is also proved by mathematical induction. The proposition is obviously true for $n=1$. Assume that the proposition is true for $n=m$. It is sufficient to prove that, for all $x_{j} \leq t_{j}, j=1, \ldots, m$

$$
F_{X_{m+1} \mid \bigcap_{j=1}^{m} X_{j}=x_{j}}\left(x_{m+1}\right) \geq F_{X_{m+1} \mid \bigcap_{j=1}^{m} X_{j}=t_{j}}\left(x_{m+1}\right) .
$$


If $\mathbf{t}_{m+1} \in R_{\mathbf{X}}(\mathbf{x})$, then, for $s=1, \ldots, m+1$, it is held that

$$
t_{s} \geq F_{X_{s} \mid \bigcap_{j=1}^{s-1} X_{j}=t_{j}}^{-}\left[F_{X_{s} \mid \cap_{j=1}^{s-1} X_{j}=x_{j}}\left(x_{s}\right)\right] .
$$

In particular, if

$$
t_{m+1}=F_{X_{m+1} \mid \bigcap_{j=1}^{m} X_{j}=t_{j}}^{-}\left[F_{X_{m+1} \mid \bigcap_{j=1}^{m} X_{j}=x_{j}}\left(x_{m}\right)\right],
$$

given that $R_{\mathbf{X}}(\mathbf{x}) \subset C(\mathbf{x})$ for all $\mathbf{x} \in \mathbb{R}^{n}$, it holds that

$$
F_{X_{m+1} \mid \bigcap_{j=1}^{m} X_{j}=t_{j}}^{-}\left[F_{X_{m+1} \mid \bigcap_{j=1}^{m} X_{j}=x_{j}}\left(x_{m}\right)\right] \geq x_{m+1} .
$$

Now, from the fact that $F_{X_{m+1} \mid \cap_{j=1}^{m} X_{j}=x_{j}}(x)$ is an increasing function in $x$, by solving the inequality (3), the inequality (2) is obtained.

Theorem 2. $\mathbf{X}$ is a CDS random vector if and only if $C(\mathbf{x}) \cap \operatorname{Supp}(\mathbf{X}) \subset R_{\mathbf{X}}(\mathbf{x})$ for all $\mathbf{x} \in \mathbb{R}^{n}$

Proof. First, we prove the sufficient conditions. The proof will be obtained by mathematical induction. The proposition is obviously true for $n=1$. Assume that the proposition is true for $n=m$. Now we need to show that it is true for $n=m+1$. If $\mathbf{t}_{m+1} \in C\left(\mathbf{x}_{m+1}\right) \cap \operatorname{Supp}(\mathbf{X})$, then $\mathbf{t}_{m+1} \geq \mathbf{x}_{m+1}$. Since $\mathbf{X}$ is CDS, it follows that, for all $x_{j} \leq t_{j}, j=1, \ldots, m$

$$
F_{X_{m+1} \mid \bigcap_{j=1}^{m} X_{j}=t_{j}}\left(x_{m+1}\right) \geq F_{X_{m+1} \mid \bigcap_{j=1}^{m} X_{j}=x_{j}}\left(x_{m+1}\right) .
$$

On the other hand, given that $F_{X_{m+1} \mid \cap_{j=1}^{m} X_{j}=t_{j}}(x)$ is increasing in $x$,

$$
F_{X_{m+1} \mid \bigcap_{j=1}^{m} X_{j}=t_{j}}\left(t_{m+1}\right) \geq F_{X_{m+1} \mid \bigcap_{j=1}^{m} X_{j}=t_{j}}\left(x_{m+1}\right) .
$$

From (4) and (5) it holds that

$$
F_{X_{m+1} \mid \cap_{j=1}^{m} X_{j}=t_{j}}\left(t_{m+1}\right) \geq F_{X_{m+1} \mid \bigcap_{j=1}^{m} X_{j}=x_{j}}\left(x_{m+1}\right) .
$$

Given that $F_{X_{m+1} \mid \cap_{j=1}^{m} X_{j}=t_{j}}^{-}(u)$ is an increasing function in $u$, then

$$
t_{m+1} \geq F_{X_{m+1} \mid \bigcap_{j=1}^{m} X_{j}=t_{j}}^{-}\left[F_{X_{m+1} \mid \bigcap_{j=1}^{m} X_{j}=x_{j}}\left(x_{m+1}\right)\right] .
$$

Therefore, $\mathbf{t}_{m+1} \in R_{\mathbf{X}}(\mathbf{x})$.

The necessary condition is also proved by mathematical induction. The proposition is obviously true for $n=1$. Assume that the proposition is true for $n=m$, then it is sufficient to prove that, for all $x_{j} \leq t_{j}, j=1, \ldots, m$, it holds

$$
F_{X_{m+1} \mid \bigcap_{j=1}^{m} X_{j}=t_{j}}\left(x_{m+1}\right) \geq F_{X_{m+1} \mid \bigcap_{j=1}^{m} X_{j}=x_{j}}\left(x_{m+1}\right) .
$$

If $\mathbf{t}_{m+1} \in C(\mathbf{x}) \cap \operatorname{Supp}(\mathbf{x}) \subset R_{\mathbf{X}}(\mathbf{x})$, then

$$
t_{s} \geq F_{X_{s} \mid \bigcap_{j=1}^{s-1} X_{j}=t_{j}}^{-}\left[F_{X_{s} \mid \bigcap_{j=1}^{s-1} X_{j}=x_{j}}\left(x_{s}\right)\right]
$$

and $t_{s} \geq x_{s}$, for $s=1, \ldots, m+1$. In particular, if $t_{m+1}=$ $x_{m+1}$, it holds that

$$
x_{m+1} \geq F_{X_{m+1} \mid \bigcap_{j=1}^{m} X_{j}=t_{j}}^{-}\left[F_{X_{m+1} \mid \bigcap_{j=1}^{m} X_{j}=x_{j}}\left(x_{m+1}\right)\right],
$$

and, given that $F_{X_{m+1} \mid \cap_{j=1}^{m} X_{j}=t_{j}}(x)$ is an increasing function in $x$, the inequality (6) is obtained. Thus, $\mathbf{X}$ is CDS.

Now, the monotonicity of the $\widehat{\mathbf{x}}(\mathbf{u})$ is characterized by the CIS or CDS property of the distribution function of $\mathbf{X}$. Let $\phi_{\mathbf{X}}:[0,1]^{n} \rightarrow \mathbb{R}^{n}$ be such that $\phi_{\mathbf{X}}(\mathbf{u})=\widehat{\mathbf{x}}(\mathbf{u})$ and $\phi_{\mathbf{X}}^{-1}$ its corresponding inverse function.

Theorem 3. $\mathbf{X}$ is a CIS random vector if and only if $\phi_{\mathbf{X}}$ is increasing.

Proof. For the sufficient condition, see Rubinstein et al. (1985).

The necessary condition will be by mathematical induction. From Theorem 1, it is sufficient to prove that $R_{\mathbf{X}}(\mathbf{x}) \subset C(\mathbf{x})$. The statement is obviously true for $n=1$. Assume that the proposition is true for $n=m$. Let $\mathbf{t}_{m+1} \in R_{\mathbf{X}}(\mathbf{x})$, that is,

$$
t_{s} \geq F_{X_{s} \mid \bigcap_{j=1}^{s-1} X_{j}=t_{j}}^{-}\left[F_{X_{s} \mid \bigcap_{j=1}^{s-1} X_{j}=x_{j}}\left(x_{s}\right)\right]
$$

for $s=1, \ldots, m+1$. In particular, for $s=m+1$, it holds

$$
t_{m+1} \geq F_{X_{m+1} \mid \bigcap_{j=1}^{m} X_{j}=t_{j}}^{-}\left[F_{X_{m+1} \mid \bigcap_{j=1}^{m} X_{j}=x_{j}}\left(x_{m+1}\right)\right]
$$

and, given that $F_{X_{m+1} \mid} \mid \bigcap_{j=1}^{m} X_{j}=t_{j}(x)$ is increasing in $x$, it follows

$$
F_{X_{m+1} \mid \bigcap_{j=1}^{m} X_{j}=t_{j}}\left(t_{m+1}\right) \geq F_{X_{m+1} \mid \bigcap_{j=1}^{m} X_{j}=x_{j}}\left(x_{m+1}\right) .
$$

Now, let $t_{j}=\widehat{x}\left(\mathbf{v}_{j}\right), x_{j}=\widehat{x}\left(\mathbf{u}_{j}\right)$ for $j=1, \ldots, m$ and $v_{m+1}=F_{X_{m+1} \mid \bigcap_{j=1}^{m} X_{j}=t_{j}}\left(t_{m+1}\right)$ and $u_{m+1}=$ $F_{X_{m+1} \mid \bigcap_{j=1}^{m} X_{j}=x_{j}}\left(x_{m+1}\right)$. Then, by the hypothesis, $\widehat{x}\left(\mathbf{v}_{m+1}\right) \geq \widehat{x}\left(\mathbf{u}_{m+1}\right)$, that is

$\mathrm{F}_{X_{m+1} \mid \bigcap_{j=1}^{m} X_{j}=\widehat{x}\left(\mathbf{v}_{j}\right)}^{-}\left[F_{X_{m+1} \mid \bigcap_{j=1}^{m} X_{j}=\widehat{x}\left(\mathbf{v}_{j}\right)}\left(t_{m+1}\right)\right]$ $\geq F_{X_{m+1} \mid \bigcap_{j=1}^{m} X_{j}=\widehat{x}\left(\mathbf{u}_{j}\right)}\left[F_{X_{m+1} \mid \bigcap_{j=1}^{m} X_{j}=\widehat{x}\left(\mathbf{u}_{j}\right)}\left(x_{m+1}\right)\right]$.

Therefore, $t_{m+1} \geq x_{m+1}$ and the result is obtained.

Note that this theorem shows that the CIQ property, which was given in Belzunce et al. (2008), is equivalent to the CIS property. Thus, as a consequence, all the multivariate dispersive orders considered in Belzunce et al. (2008), are equivalent for CIS vectors having a common copula. 
Theorem 4. $\mathbf{X}$ is a $C D S$ random vector if and only if $\phi_{\mathbf{X}}^{-1}$ in increasing.

Proof. Suppose that $\mathbf{X}$ is a CDS random vector. The proof will be by mathematical induction. The proposition is obviously true for $n=1$. Assume that the proposition is true for $n=m$. If $\mathbf{X}$ is a CDS random vector, then

$F_{X_{m+1} \mid \bigcap_{j=1}^{m} X_{j}=\widehat{\mathbf{x}}\left(\mathbf{u}_{j}\right)}^{-}\left(u_{m+1}\right) \geq F_{X_{m+1} \mid \bigcap_{j=1}^{m} X_{j}=\widehat{\mathbf{x}}\left(\mathbf{v}_{j}\right)}^{-}\left(u_{m+1}\right)$

for all $\mathbf{u}_{j} \leq \mathbf{v}_{j}, j=1, \ldots, m$. If $\widehat{\mathbf{x}}\left(\mathbf{u}_{m+1}\right) \leq \widehat{\mathbf{x}}\left(\mathbf{v}_{m+1}\right)$, then by definition

$F_{X_{m+1} \mid \bigcap_{j=1}^{m} X_{j}=\widehat{x}\left(\mathbf{u}_{j}\right)}^{-}\left(u_{m+1}\right) \leq F_{X_{m+1} \mid \bigcap_{j=1}^{m} X_{j}=\widehat{x}\left(\mathbf{v}_{j}\right)}^{-}\left(v_{m+1}\right)$.

Finally, from (7) and (8) and given that $F_{X_{m+1} \mid \bigcap_{j=1}^{m} X_{j}=\widehat{x}\left(\mathbf{v}_{j}\right)}^{-}(u)$ is increasing in $u$, it follows that $v_{m+1} \geq u_{m+1}$ and the result is obtained.

Conversely, suppose that $\widehat{\mathbf{x}}\left(\mathbf{u}_{m+1}\right) \leq \widehat{\mathbf{x}}\left(\mathbf{v}_{m+1}\right)$ implies $\mathbf{u}_{m+1} \leq \mathbf{v}_{m+1}$. In the light of Theorem 2, it is sufficient to prove that $C(\mathbf{x}) \subset R_{\mathbf{X}}(\mathbf{x})$ for all $\mathbf{x} \in \mathbb{R}^{m}$.

Let $\mathbf{t}_{m+1} \in C(\mathbf{x})$, that is,

$$
t_{s} \geq x_{s} \text { for } s=1, \ldots, m+1 .
$$

In particular, for $s=m+1$ and from the regularity conditions, the inequality (9) is equivalent to $\mathrm{F}_{X_{m+1} \mid \bigcap_{j=1}^{m} X_{j}=t_{j}}^{-}\left[F_{X_{m+1}} \mid \bigcap_{j=1}^{m} X_{j}=t_{j}\left(t_{m+1}\right)\right] \geq$ $F_{X_{m+1} \mid \bigcap_{j=1}^{m} X_{j}=x_{j}}^{-}\left[F_{X_{m+1} \mid \bigcap_{j=1}^{m} X_{j}=x_{j}}\left(x_{m+1}\right)\right]$.

Now, if for $j=1, \ldots, m$ it is $t_{j}=\widehat{x}\left(\mathbf{v}_{j}\right)$ and $x_{j}=\widehat{x}\left(\mathbf{u}_{j}\right)$, and

$$
\begin{aligned}
& v_{m+1}=F_{X_{m+1} \mid \bigcap_{j=1}^{m} X_{j}=t_{j}}\left(t_{m+1}\right), \\
& u_{m+1}=F_{X_{m+1} \mid \bigcap_{j=1}^{m} X_{j}=x_{j}}\left(x_{m+1}\right),
\end{aligned}
$$

then the above inequality can be rewritten as

$$
F_{X_{m+1} \mid \bigcap_{j=1}^{m} X_{j}=\widehat{x}\left(\mathbf{v}_{j}\right)}^{-}\left(v_{m+1}\right) \geq F_{X_{m+1} \mid \bigcap_{j=1}^{m} X_{j}=\widehat{x}\left(\mathbf{u}_{j}\right)}^{-}\left(u_{m+1}\right) .
$$

Moreover, by the hypothesis and the inequality (10), $v_{m+1} \geq u_{m+1}$ holds. Consequently, given that $F_{X_{m+1} \mid \bigcap_{j=1}^{m} X_{j}=t_{j}}(x)$ is increasing in $x$, it follows that

$$
t_{m+1} \geq F_{X_{m+1} \mid \bigcap_{j=1}^{m} X_{j}=t_{j}}^{-}\left[F_{X_{m+1} \mid \bigcap_{j=1}^{m} X_{j}=x_{j}}\left(x_{m+1}\right)\right] .
$$

Therefore, $\mathbf{t}_{m+1} \in R_{\mathbf{X}}(\mathbf{x})$ and the result is obtained.

\section{The multivariate excess wealth function}

In this section, the multivariate excess wealth function is defined following the same steps given for definition in the univariate case in Fernández-Ponce et al. (1996). One purpose is to generalize the univariate version and to prove that it preserves some similar properties.
Definition 3. Let $\mathbf{X}$ be a nonnegative random vector. Then the multiple expectation associated to $\mathbf{X}$, when it exists, is defined as the real value

$$
\bar{\mu}_{\mathbf{X}}=\int_{\operatorname{Supp}(\mathbf{X})} P\left[\mathbf{X} \in R_{\mathbf{X}}(\mathbf{t})\right] d \mathbf{t} .
$$

Remark 2. Let $\mathbf{X}=\left(X_{1}, X_{2}\right)$ be a bivariate random variable. Then under straightforward calculations is easily obtained by using (1) that

$$
\bar{\mu}_{\mathbf{X}}=\nu_{\mathbf{X}}-\int_{F_{X 1}^{-}(0)}^{+\infty} \bar{F}_{1}(t) F_{X_{2} \mid X_{1}=t}^{-}(0) d t,
$$

where $\nu_{\mathbf{X}}=\int_{F_{X_{1}}^{-}(0)}^{\infty} \bar{F}_{1}(t) \mathbf{E}\left[X_{2} \mid X_{1}=t\right] d t$. Particularly, if $\mathbf{X}$ represents a non-negative lifetime random variable then

$$
\bar{\mu}_{\mathbf{X}} \leq \nu_{\mathbf{X}}
$$

If $\operatorname{Supp}(\mathbf{X})=[0,+\infty)^{2}$, then $\bar{\mu}_{\mathbf{X}}=\nu_{\mathbf{X}}$ holds. Furthermore, if the components are independent random variables, then the multiple expectation coincides with the product of the marginal expectations.

The value of $\nu_{\mathbf{X}}$ in the bivariate case can be easily obtained if the first component has an exponential distribution. This fact is proved in the following proposition.

Proposition 2. Let $\mathbf{X}=\left(X_{1}, X_{2}\right)$ be a couple of random lifetimes, and let $X_{1}$ have an exponential distribution. Then $\nu_{\mathbf{X}}=\mathbf{E}\left[X_{1}\right] \cdot \mathbf{E}\left[X_{2}\right]$.

Proof. Given that $X_{1}$ has an exponential distribution, it holds that $X_{1}={ }_{s t} X_{1}^{e}$, where $X_{1}^{e}$ is the lifetime having density function $f_{X_{1}^{e}}(t)=\bar{F}_{X_{1}}(t) / \mathbf{E}\left[X_{1}\right], t \geq 0$.

It can also be noted that $\mathbf{E}\left[X_{2}\right]=\int_{0}^{+\infty} \mathbf{E}\left[X_{2} \mid X_{1}=\right.$ $t] d F_{X_{1}}(t)$, so that

$$
\int_{0}^{+\infty}\left(\mathbf{E}\left[X_{2} \mid X_{1}=t\right]-\mathbf{E}\left[X_{2}\right]\right) d F_{X_{1}}(t)=0 .
$$

Now, let the function $\varphi(t)$ defined as $\varphi(t)=\mathbf{E}\left[X_{2} \mid X_{1}=\right.$ $t]-\mathbf{E}\left[X_{2}\right]$. By using (11), it holds that $\mathbf{E}\left[\varphi\left(X_{1}\right)\right]=0$ and by taking into account that $X_{1}={ }_{s t} X_{1}^{e}$, it follows

$$
\begin{aligned}
0 & =\mathbf{E}\left[\varphi\left(X_{1}\right)\right]=\mathbf{E}\left[\varphi\left(X_{1}^{e}\right)\right] \\
& =\int_{0}^{+\infty}\left(\mathbf{E}\left[X_{2} \mid X_{1}=t\right]-\mathbf{E}\left[X_{2}\right]\right) d F_{X_{1}^{e}}(t) \\
& =\int_{0}^{+\infty}\left(\mathbf{E}\left[X_{2} \mid X_{1}=t\right]-\mathbf{E}\left[X_{2}\right]\right) \frac{\bar{F}_{X_{1}}(t)}{\mathbf{E}\left[X_{1}\right]} d t
\end{aligned}
$$

By using the previous inequality jointly with the fact that $\nu_{\mathbf{X}}=\int_{0}^{+\infty} \mathbf{E}\left[X_{2} \mid X_{1}=t\right] \bar{F}_{X_{1}}(t) d t$ and $\mathbf{E}\left[X_{1}\right]>0$, it is immediately obtained that

$$
\begin{aligned}
\nu_{\mathbf{X}} & =\int_{0}^{+\infty} \mathbf{E}\left[X_{2} \mid X_{1}=t\right] \bar{F}_{X_{1}}(t) d t \\
& =\int_{0}^{+\infty} \mathbf{E}\left[X_{2}\right] \bar{F}_{X_{1}}(t) d t=\mathbf{E}\left[X_{1}\right] \mathbf{E}\left[X_{2}\right]
\end{aligned}
$$


Definition 4. Let $\mathbf{X}$ be a nonnegative random vector with finite multiple expectation. The multivariate excess wealth function associated to $\mathbf{X}$ is defined as

$$
S_{\mathbf{X}}^{+}(\mathbf{u})=\int_{R_{\mathbf{X}}[\hat{\mathbf{x}}(\mathbf{u})]} P\left[\mathbf{X} \in R_{\mathbf{X}}(\mathbf{t})\right] d \mathbf{t}, \quad \mathbf{u} \in[0,1)^{n} .
$$

Several interesting properties for the multivariate excess function can be shown as in the univariate case.

Proposition 3. Let $\mathbf{X}$ be a nonnegative random vector with finite multiple expectation. Then

i) $S_{\mathbf{X}}^{+}(\mathbf{u})$ is a decreasing and a shift invariant function.

ii) $0 \leq S_{\mathbf{X}}^{+}(\mathbf{u}) \leq S_{\mathbf{X}}^{+}(\mathbf{0})=\bar{\mu}_{\mathbf{X}}$ for all $\mathbf{u}$ in $[0,1)^{n}$.

iii) If the components of $\mathbf{X}$ are independent, then

$$
S_{\mathbf{X}}^{+}(\mathbf{u})=\prod_{i=1}^{n} S_{X_{i}}^{+}\left(u_{i}\right)
$$

Proof. Trivial and therefore omitted.

Particularly, it is interesting to obtain an expression for the bivariate excess wealth function easier to hand. Let $\mathbf{X}=$ $\left(X_{1}, X_{2}\right)$ be a bi-dimensional random vector satisfying the $\mathrm{RC}$ conditions with density function $f_{\mathbf{X}}\left(x_{1}, x_{2}\right)$. Obviously, it holds that

$$
\widehat{\mathbf{x}}\left(u_{1}, u_{2}\right)=\left(F_{X_{1}}^{-}\left(u_{1}\right), F_{X_{2} \mid X_{1}=F_{X_{1}}^{-}\left(u_{1}\right)}^{-}\left(u_{2}\right)\right)
$$

and $\mathbf{x} \in \mathbf{R}_{\mathbf{X}}\left(\widehat{\mathbf{x}}\left(\mathbf{u}_{\mathbf{1}}, \mathbf{u}_{\mathbf{2}}\right)\right)$ if

$$
x_{1} \geq F_{X_{1}}^{-}\left(u_{1}\right) \text { and } x_{2} \geq F_{X_{2} \mid X_{1}=x_{1}}^{-}\left(u_{2}\right) \text {. }
$$

Consequently, by using (1) the bivariate excess wealth function can be expressed as

$$
S_{\mathbf{X}}^{+}\left(u_{1}, u_{2}\right)=\int_{F_{X_{1}}^{-}\left(u_{1}\right)}^{\infty} \bar{F}_{X_{1}}\left(t_{1}\right) \cdot S_{X_{2} \mid X_{1}=t_{1}}^{+}\left(u_{2}\right) d t_{1}
$$

Differentiating (12), it is easily shown that

$$
\frac{\partial S_{\mathbf{X}}^{+}\left(u_{1}, u_{2}\right)}{\partial u_{2} \partial u_{1}}=\frac{P\left(\mathbf{X} \in R_{\mathbf{X}}[\hat{\mathbf{x}}(\mathbf{u})]\right)}{f_{\mathbf{X}}(\widehat{\mathbf{x}}(\mathbf{u}))}
$$

for all $\left(u_{1}, u_{2}\right)$ in $(0,1)^{2}$.

\section{The multivariate excess wealth ordering}

In this section the multivariate excess wealth ordering is defined and some of its properties are proved.

Definition 5. Let $\mathbf{X}$ and $\mathbf{Y}$ be two nonnegative random vectors with finite multiple expectations. $\mathbf{X}$ is said to be smaller than $\mathbf{Y}$ in the sense of multivariate excess wealth order (denoted $\mathbf{X} \leq_{e w} \mathbf{Y}$ ) if

$$
S_{\mathbf{X}}^{+}(\mathbf{u}) \leq S_{\mathbf{Y}}^{+}(\mathbf{u}) \text { for all } \mathbf{u} \text { in }(0,1)^{n}
$$

Note that this ordering is the multivariate version of the excess wealth ordering which was studied in Shaked and Shanthikumar (1998) and Fernández-Ponce et al. (1998).

Example 1. Let $\mathbf{X}$ and $\mathbf{Y}$ have a bivariate Pareto distribution with parameters $\theta_{1}, \theta_{2}, a$ and $\theta_{1}^{\prime}, \theta_{2}^{\prime}, a^{\prime}$, respectively. If $2<a^{\prime} \leq a$ and $\theta_{i} \leq \theta_{i}^{\prime}$ for $i=1,2$, then $\mathbf{X} \leq_{e w} \mathbf{Y}$.

In fact, if $X$ has a joint Pareto density given by

$$
f\left(t_{1}, t_{2}\right)=\frac{a(a+1)}{\theta_{1} \theta_{2}}\left(\frac{t_{1}}{\theta_{1}}+\frac{t_{2}}{\theta_{2}}-1\right)^{-(a+2)},
$$

with, $a>0, t_{i} \geq \theta_{i}>0$, easy calculations show that, for $t_{i} \geq \theta_{i}$,

$$
\bar{F}_{X_{2} \mid X_{1}=t_{1}}\left(t_{2}\right)=\frac{t_{1}^{(a+1)}}{\theta_{1}^{(a+1)}}\left(\frac{t_{1}}{\theta_{1}}+\frac{t_{2}}{\theta_{2}}-1\right)^{-(a+1)} .
$$

The excess-wealth function of $X_{2} \mid X_{1}=t_{1}$ has the following expression:

$$
\begin{aligned}
& S_{X_{2} \mid X_{1}=t_{1}}^{+}\left(u_{2}\right)=\int_{F_{X_{2} \mid X_{1}=t_{1}}^{+}\left(u_{2}\right)}^{+\infty} \bar{F}_{X_{2} \mid X_{1}=t_{1}}\left(t_{2}\right) d t_{2} \\
& =\int_{F_{X_{2} \mid X_{1}=t_{1}}^{+}\left(u_{2}\right)}^{+\infty} \frac{t_{1}^{(a+1)}}{\theta_{1}^{(a+1)}}\left(\frac{t_{1}}{\theta_{1}}+\frac{t_{2}}{\theta_{2}}-1\right)^{-(a+1)} d t_{1} \\
& =\frac{t_{1} \theta_{2}}{a \theta_{1}}\left(1-u_{2}\right)^{\frac{a}{a+1}} .
\end{aligned}
$$

Therefore, by using (4), the bivariate excess-wealth for the vector $\mathbf{X}$ is given by

$$
\begin{aligned}
S_{\mathbf{X}}\left(u_{1}, u_{2}\right) & =\int_{F_{X_{1}}^{-}\left(u_{1}\right)}^{+\infty} \bar{F}_{X_{1}}\left(t_{1}\right) S_{X_{2} \mid X_{1}=t_{1}}^{+}\left(u_{2}\right) d t_{1} \\
& =\int_{F_{X_{1}}^{-}\left(u_{1}\right)}^{\infty} \theta_{1}^{a} t_{1}^{-a} \frac{t_{1} \theta_{2}}{a \theta_{1}}\left(1-u_{2}\right)^{\frac{a}{a+1}} d t_{1} \\
& =\frac{\theta_{2} \theta_{1}\left(1-u_{2}\right)^{\frac{a}{a+1}}\left(1-u_{1}\right)^{\frac{a-2}{a}}}{a(a-2)},
\end{aligned}
$$

for $a>2$. It is easily seen that the functions $h(a)=$ $\left(1-u_{2}\right)^{\frac{a}{a+1}}\left(1-u_{1}\right)^{\frac{a-2}{a}}$ and $g(a)=\frac{1}{a(a-2)}$ are decreasing in a. So, for $2<a^{\prime} \leq a$ and $\theta_{i} \leq \theta_{i}^{\prime} i=1,2$, it follows that

$$
\begin{aligned}
& \frac{\theta_{2} \theta_{1}\left(1-u_{2}\right)^{\frac{a}{a+1}}\left(1-u_{1}\right)^{\frac{a-2}{a}}}{a(a-2)} \leq \\
& \frac{\theta_{2}^{\prime} \theta_{1}^{\prime}\left(1-u_{2}\right)^{\frac{a^{\prime}}{a^{\prime}+1}}\left(1-u_{1}\right)^{\frac{a^{\prime}-2}{a^{\prime}}}}{a^{\prime}\left(a^{\prime}-2\right)}
\end{aligned}
$$


that is, $\mathbf{X} \leq_{e w} \mathbf{Y}$.

It is easy to prove the following closure results. The notation $\sim_{e w}$ means that both $\mathbf{X} \leq_{e w} \mathbf{Y}$ and $\mathbf{Y} \leq_{e w} \mathbf{X}$ hold.

Theorem 5. i) Let $\mathbf{X}^{(1)}, \ldots, \mathbf{X}^{(m)}$ be a set of independent random vectors and let $\mathbf{Y}^{(1)}, \ldots, \mathbf{Y}^{(m)}$ be another set of independent random vectors. If $\mathbf{X}^{(i)} \leq_{\text {ew }}$ $\mathbf{Y}^{(i)}$ for $i=1, \ldots, m$, then $\left(\mathbf{X}^{(1)}, \ldots, \mathbf{X}^{(m)}\right) \leq_{e w}$ $\left(\mathbf{Y}^{(1)}, \ldots, \mathbf{Y}^{(m)}\right)$.

ii) Let $\left\{\mathbf{X}^{(j)}: j=1,2 \ldots\right\}$ and $\left\{\mathbf{Y}^{(j)}: j=1,2, \ldots\right\}$ be two sequences of random vectors such that $\mathbf{X}^{(j)} \rightarrow_{\text {st }}$ $\mathbf{X}$ and $\mathbf{Y}^{(j)} \rightarrow_{s t} \mathbf{Y}$ as $j \rightarrow \infty$, where $\rightarrow_{\text {st }}$ denotes convergence in distribution. If $\mathbf{X}^{(j)} \leq_{e w}$ $\mathbf{Y}^{(j)}$ for every $j=1,2, \ldots$, then $\mathbf{X} \leq_{e w} \mathbf{Y}$.

Proof. i) It is trivial by using Definition 5 and iii) of the Proposition 3.

ii) It is easy to show that if $\mathbf{X}^{(j)} \rightarrow_{s t} \mathbf{X}$ then $\widehat{\mathbf{x}}^{(j)}(\mathbf{u}) \rightarrow \widehat{\mathbf{x}}(\mathbf{u})$ and $R_{\mathbf{X}^{(j)}}\left[\widehat{\mathbf{x}}^{(j)}(\mathbf{u})\right] \rightarrow$ $R_{\mathbf{X}}[\widehat{\mathbf{x}}(\mathbf{u})]$ for all $\mathbf{u}$ in $(0,1)^{n}$. Now by using the monotone convergence theorem and the fact that $\bar{\mu}_{\mathbf{X}^{(j)}}<+\infty$ for all $j$, it is easily shown that $S_{\mathbf{X}_{(j)}^{+}}^{+}(\mathbf{u}) \rightarrow S_{\mathbf{X}}^{+}(\mathbf{u})$. Thus the result is immediately obtained.

Some properties and results of the excess wealth order are proved for the bivariate case. These properties can be easily generalized to any dimension.

Theorem 6. Let $\mathbf{X}=\left(X_{1}, X_{2}\right)$ and $\mathbf{Y}=\left(Y_{1}, Y_{2}\right)$ be two random vectors.

1. If $\mathbf{X} \sim_{s t} \mathbf{Y}+\mathbf{c}$ then $\mathbf{X} \sim_{e w} \mathbf{Y}$.

2. If $\mathbf{X} \sim_{\text {ew }} \mathbf{Y}$ then $f[\widehat{\mathbf{x}}(\mathbf{u})]=g[\widehat{\mathbf{y}}(\mathbf{u})]$ for all $\mathbf{u}$ in $(0,1)^{2}$, where $f(\cdot)$ and $g(\cdot)$ are the corresponding density functions for $\mathbf{X}$ and $\mathbf{Y}$, respectively.

Proof. (1) Trivial, since the multivariate excess wealth function is shift invariant, as it can be easily verified.

(2) Suppose $\mathbf{X} \sim_{e w} \mathbf{Y}$, i.e., suppose it holds

$$
S_{\mathbf{X}}^{+}(\mathbf{u})=S_{\mathbf{Y}}^{+}(\mathbf{u}) \text { for all } \mathbf{u} \text { in }(0,1)^{2} .
$$

Since $\mathbf{X}$ and $\mathbf{Y}$ satisfy the regularity conditions, it follows that the quantiles $\widehat{\mathbf{x}}(\mathbf{u})$ and $\widehat{\mathbf{y}}(\mathbf{u})$ are differentiable with respect to $\mathbf{u}=\left(u_{1}, u_{2}\right)$ at each component. Consequently, by using (14) and Proposition 1, it is immediately obtained the result.

A multivariate dispersion ordering based on the standard construction was given by Fernández-Ponce and SuárezLloréns (2003). Given two $n$-dimensional random vectors $\mathbf{X}$ and $\mathbf{Y}$, we say that $\mathbf{X}$ is smaller than $\mathbf{Y}$ in the multivariate dispersion order, denoted by $\mathbf{X} \leq_{\text {disp }} \mathbf{Y}$, if, and only if,

$$
\|\hat{x}(\mathbf{v})-\hat{x}(\mathbf{u})\|_{2} \leq\|\hat{y}(\mathbf{v})-\hat{y}(\mathbf{u})\|_{2},
$$

for all $\mathbf{u}, \mathbf{v} \in[0,1]^{n}$, where $\|\cdot\|_{2}$ means the Euclidean distance.

Fernández-Ponce and Suárez-Lloréns (2003) established that the multivariate dispersion ordering is characterized by an unique expansion function which maps each quantile for one variable in the corresponding quantile for the other one. Particularly, Arias-Nicolás et al. (2005) studied the concept of multivariate dispersion order for multivariate distributions with the same dependence structure. Later on, Belzunce et al. (2008) studied a weaker multivariate dispersion ordering defined as $\mathbf{X}$ is said to be less in the conditional dispersive order than $\mathbf{Y}$, denoted by $\mathbf{X} \leq_{c-\operatorname{disp}} \mathbf{Y}$, if, and only if,

$$
\begin{aligned}
X_{1} & \leq \text { disp } \\
X_{i} \mid \bigcap_{j=1}^{i-1} X_{j}=\hat{x}_{j}\left(\mathbf{u}_{j}\right) & \leq{ }_{\text {disp }} \quad Y_{i} \mid \bigcap_{j=1}^{i-1} Y_{j}=\hat{y}_{j}\left(\mathbf{u}_{j}\right) .
\end{aligned}
$$

Now, it is proved that the multivariate conditional dispersion ordering implies the excess wealth ordering.

Theorem 7. Let $\mathbf{X}$ and $\mathbf{Y}$ be two absolutely continuous random vectors. If $\mathbf{X} \leq_{c-\text { disp }} \mathbf{Y}$ then $\mathbf{X} \leq_{\text {ew }} \mathbf{Y}$.

Proof. For simplicity, the bidimensional case is proved. This proof can easily be generalized to any dimension. By using the equality (1), the bivariate excess-wealth function of $\mathbf{X}$ can be expressed as

$S_{\mathbf{X}}^{+}(\mathbf{u})=\int_{F_{X_{1}}^{-}\left(u_{1}\right)}^{\infty} \int_{F_{X_{2} \mid X_{1}=t_{1}}^{-}}^{\infty} \bar{F}_{X_{1}}\left(t_{1}\right) \bar{F}_{X_{2} \mid X_{1}=t_{1}}\left(t_{2}\right) d t_{1} d t_{2}$.

By taking $w_{1}=F_{X_{1}}\left(t_{1}\right)$ and $w_{2}=F_{X_{2} \mid X_{1}=t_{1}}\left(t_{2}\right)$, it follows that

$$
S_{\mathbf{X}}^{+}(\mathbf{u})=\int_{u_{1}}^{1} \int_{u_{2}}^{1} \frac{\left(1-w_{1}\right)\left(1-w_{2}\right)}{f_{\mathbf{X}}(\hat{x}(\mathbf{w}))} d w_{1} d w_{2}
$$

where $f_{\mathbf{X}}(\cdot)$ is the density function of $\mathbf{X}$. Similarly,

$$
S_{\mathbf{Y}}^{+}(\mathbf{u})=\int_{u_{1}}^{1} \int_{u_{2}}^{1} \frac{\left(1-w_{1}\right)\left(1-w_{2}\right)}{f_{\mathbf{Y}}(\hat{y}(\mathbf{w}))} d w_{1} d w_{2}
$$

where $f_{\mathbf{Y}}(\cdot)$ is the density function of $\mathbf{Y}$.

It is clear that if $\mathbf{X} \leq_{c-\operatorname{disp}} \mathbf{Y}$, then $f_{\mathbf{X}}(\hat{x}(\mathbf{w})) \geq$ $f_{\mathbf{Y}}(\hat{y}(\mathbf{w})) \quad \forall \quad \mathbf{w} \in[0,1]^{2}$ (see Belzunce et al., 2008).

Therefore, the result is immediately obtained.

An example where two random vectors are ordered in excess wealth, but not in multivariate dispersion sense, is given here. 
Example 2. Suppose that $\mathbf{X}=\left(X_{1}, X_{2}\right)$ is a bivariate exponential random vector such that $X_{1}$ and $X_{2}$ are independent, $X_{1} \quad \sim_{s t} \operatorname{Exp}(2)$ and $X_{2} \sim_{s t} \operatorname{Exp}(1) . \quad$ Similarly, assume that $\mathbf{Y}=\left(Y_{1}, Y_{2}\right)$ is a bivariate exponential random vector such that $Y_{1}$ and $Y_{2}$ are independent with $Y_{1} \sim_{s t} \operatorname{Exp}(1 / 3)$ and $Y_{2} \sim_{s t} \operatorname{Exp}(3)$. Consequently, it holds that

$$
X_{1}<_{\text {disp }} Y_{1} \text { and } Y_{2}<_{\text {disp }} X_{2}
$$

which implies that $\mathbf{X} \nless_{c-\text { disp }} \mathbf{Y}$. Furthermore, by using iii) of Proposition 3, it holds

$$
\begin{aligned}
S_{\mathbf{X}}^{+}\left(u_{1}, u_{2}\right) & =\frac{1}{2}\left(1-u_{1}\right)\left(1-u_{2}\right) \\
& \leq\left(1-u_{1}\right)\left(1-u_{2}\right)=S_{\mathbf{Y}}^{+}\left(u_{1}, u_{2}\right)
\end{aligned}
$$

for all $\left(u_{1}, u_{2}\right)$ in $(0,1)^{2}$, i.e. $\mathbf{X} \leq_{e w} \mathbf{Y}$.

The following result describes conditions under which the multivariate excess wealth order holds. Some previous definitions are necessary. Let $X$ and $Y$ be two univariate random variables with excess wealth function $S_{X}^{+}(u)$ and $S_{Y}^{+}(u)$, respectively.

Definition 6. Let $X$ and $Y$ be two univariate random variables. $X$ is said to be smaller than $Y$ in relative excess wealth sense (denoted $X \leq_{\text {rew }} Y$ ) if

$$
\frac{S_{X}^{+}(v)}{S_{X}^{+}(u)} \leq \frac{S_{Y}^{+}(v)}{S_{Y}^{+}(u)} \text { for all } 0<u \leq v \leq 1
$$

Note that, when the supports of $X$ and $Y$ are $[0,+\infty)$, this ordering is equivalent to the DMRL ordering which was defined in Kochar and Wiens (1987) and further characterized in Fernández-Ponce, et al. (1996).

Definition 7. Let $X$ and $Y$ be two univariate random variables. $X$ is said to be smaller than $Y$ in wealth sense (denoted $X \leq_{W} Y$ ) if

$$
X \leq_{S T} Y \text { and } X \leq_{\text {rew }} Y .
$$

Definition 8. Let $\mathbf{X}=\left(X_{1}, X_{2}\right)$ be a bidimensional random vector. $\mathbf{X}$ is said to be conditional increasing in excess wealth $(\mathbf{X} \in C I E W)$ if

$$
X_{2}\left|X_{1}=t_{1} \leq_{e w} X_{2}\right| X_{1}=t_{2} \text { for all } t_{1} \leq t_{2} .
$$

Recall that a distribution function $\mathrm{F}$ is said to be of powerlaw tail or Pareto type tail if, for $x \rightarrow+\infty$,

$$
1-F(x) \sim K x^{-\mu}
$$

where $K$ is a positive normalization constant or a slowly varying function. The exponent $\mu>0$ is called the tail exponent of the distribution.
Proposition 4. Let $\mathbf{X}=\left(X_{1}, X_{2}\right)$ be a bidimensional random vector. If $\frac{d F_{X_{2} \mid X_{1}=t}^{-}(u)}{d t}$ is an increasing function in $u$ and $X_{2} \mid X_{1}=t$ is a power-law tail with exponent equals to one, for all $t$, then $\mathbf{X} \in C I E W$.

Proof. By using an expression for the right-spread function which can be seen in Fernández-Ponce et al. (1996), it is easy to obtain that

$$
S_{X}^{+}(u)=\int_{u}^{1}(1-\omega) d F_{X}^{-}(\omega) .
$$

Now, integrating by parts and using the fact that the random variable $X \sim_{S T} X_{2} \mid X_{1}=t$ is a power-law tail with exponent equals to one, it is held

$$
\frac{d S_{X_{2} \mid X_{1}=t}^{+}(u)}{d t}=\int_{u}^{1}\left(\frac{d F_{X_{2} \mid X_{1}=t}^{-}(\omega)}{d t}-\frac{d F_{X_{2} \mid X_{1}=t}^{-}(u)}{d t}\right) d \omega .
$$

and the result is immediately obtained.

Theorem 8. Let $\mathbf{X}=\left(X_{1}, X_{2}\right)$ and $\mathbf{Y}=\left(Y_{1}, Y_{2}\right)$ be two bidimensional random vectors. If it is held that

i) $X_{1} \leq_{W} Y_{1}$,

ii) $X_{2}\left|X_{1}=t \leq_{\text {ew }} Y_{2}\right| Y_{1}=t$ for all $t \in \mathbb{R}$,

iii) $\mathbf{X} \in C I E W$ or $\mathbf{Y} \in C I E W$

then $\mathbf{X} \leq_{e w} \mathbf{Y}$.

Proof. Assume that $\mathbf{Y} \in \mathrm{CIEW}$. By using (ii), it is obtained that

$$
S_{\mathbf{X}}^{+}\left(u_{1}, u_{2}\right) \leq \int_{F_{1}^{-}\left(u_{1}\right)}^{\infty} \bar{F}_{1}(t) S_{Y_{2} \mid Y_{1}=t}^{+}\left(u_{2}\right) d t
$$

Now, consider the density functions

$$
\tilde{f}_{1}(t)=\frac{\bar{F}_{1}(t)}{S_{X_{1}}^{+}\left(u_{1}\right)} I\left(t \geq F_{X_{1}}^{-}\left(u_{1}\right)\right)
$$

and

$$
\tilde{g}_{1}(t)=\frac{\bar{G}_{1}(t)}{S_{Y_{1}}^{+}\left(u_{1}\right)} I\left(t \geq F_{Y_{1}}^{-}\left(u_{1}\right)\right) .
$$

The corresponding distribution functions are

$$
\tilde{F}_{1}(x)=\left(1-\frac{S_{X_{1}}^{+}\left(F_{1}(x)\right)}{S_{X_{1}}^{+}\left(u_{1}\right)}\right) I\left(t \geq F_{X_{1}}^{-}\left(u_{1}\right)\right)
$$

and

$$
\tilde{G}_{1}(x)=\left(1-\frac{S_{Y_{1}}^{+}\left(G_{1}(x)\right)}{S_{Y_{1}}^{+}\left(u_{1}\right)}\right) I\left(t \geq F_{Y_{1}}^{-}\left(u_{1}\right)\right) .
$$

By taking into account $(i)$, we obtain that $\tilde{F}_{1} \leq_{S T} \tilde{G}_{1}$. Thus, 


$$
\begin{aligned}
& \int_{0}^{\infty} S_{X_{1}}^{+}\left(u_{1}\right) S_{Y_{2} \mid Y_{1}=t}^{+}\left(u_{2}\right) d \tilde{F}_{1}(t) \leq \\
& \int_{0}^{\infty} S_{Y_{1}}^{+}\left(u_{1}\right) S_{Y_{2} \mid Y_{1}=t}^{+}\left(u_{2}\right) d \tilde{G}_{1}(t)=S_{\mathbf{Y}}^{+}\left(u_{1}, u_{2}\right)
\end{aligned}
$$

and the result is obtained. The statement can be shown in a similar manner if $\mathbf{X} \in \mathrm{CIEW}$ is assumed.

Example 3. Assume that $X_{1}$ and $X_{2}$ are independent random variables with exponential distributions whose means are equal to $\lambda_{1}$ (i.e. $X_{i} \sim \operatorname{Exp}\left(\lambda_{1}\right)$ for $\left.i=1,2\right)$. Now, let $Y_{1}$ and $Y_{2}$ be two random variables such that

$Y_{1} \sim \operatorname{Exp}\left(\lambda_{2}\right)$ and $Y_{2} \mid Y_{1}=t \sim \operatorname{Exp}\left(\lambda_{2}(t+1)\right) \quad \lambda_{1} \leq \lambda_{2}$.

It is easy to verify that the random vectors $\mathbf{X}=\left(X_{1}, X_{2}\right)$ and $\mathbf{Y}=\left(Y_{1}, Y_{2}\right)$ verify the conditions of Theorem 8. Consequently, $\mathbf{X} \leq_{e w} \mathbf{Y}$.

The following result describes some necessary conditions for the excess wealth order. An immediate application, dealing with conditions for comparisons in dependence sense of random vectors, will be given next. For the statement of this result, we recall the definition of the IGFR property. Let $X$ be a nonnegative random variable with density function $f_{X}$, survival function $\bar{F}_{X}$ and failure rate function $r(x)=f_{X}(x) / \bar{F}_{X}(x)$. The generalized failure rate is defined as $R(X)=x r(x)$. If the function $R(x)$ in increasing in $x$, then it is said that $X$ has an increasing generalized failure rate (IGFR) distribution (the DGFR property can be defined analogously). Clearly, if $X$ has increasing failure rate then it is also IGFR. However, the reversed need not hold, given that there exist many distributions having decreasing failure rate which are IGFR (See Belzunce et al. , 1998, and Lariviere, 2006). Also, recall that if $\mathbf{X}$ is a random vector then its centered random vector is $\widetilde{\mathbf{X}}=\mathbf{X}-E(\mathbf{X})$.

Theorem 9. Let $\mathbf{X}=\left(X_{1}, X_{2}\right)$ and $\mathbf{Y}=\left(Y_{1}, Y_{2}\right)$ be two bidimensional random vectors and $\widetilde{\mathbf{X}}$ and $\widetilde{\mathbf{Y}}$ their corresponding centered random vectors. If it is held that

i) $X_{1} \sim_{S T} Y_{1}$

ii) $X_{1}$ has a IGFR distribution,

iii) $\operatorname{Supp}(\widetilde{\mathbf{X}}) \subseteq \operatorname{Supp}(\tilde{\mathbf{Y}})$,

iv) $\mathbf{X} \leq_{e w} \mathbf{Y}$

then $\operatorname{cov}\left(X_{1}, X_{2}\right) \leq \operatorname{cov}\left(Y_{1}, Y_{2}\right)$.

Proof. For an easier notation, $F_{1}(\cdot)$ is used to denote $F_{X_{1}}(\cdot)$ and $F_{Y_{1}}(\cdot)$. Assume first that $E\left(X_{2}\right)=E\left(Y_{2}\right)$. By taking into account that

$S_{\mathbf{X}}^{+}(u, 0)=\int_{F_{1}^{-}(u)}^{+\infty} \bar{F}_{1}(t)\left(E\left(X_{2} \mid X_{1}=t\right)-F_{X_{2} \mid X_{1}=t}^{-}(0)\right) d t$ and a similar formula is held for the random vector $\mathbf{Y}$, by using $(i v)$ it can be obtained the following inequality

$$
\begin{aligned}
& \int_{F_{1}^{-}(u)}^{+\infty} \bar{F}_{1}(t)\left(E\left(Y_{2} \mid Y_{1}=t\right)-E\left(X_{2} \mid X_{1}=t\right)\right) d t- \\
& \int_{F_{1}^{-}(u)}^{+\infty} \bar{F}_{1}(t)\left(F_{Y_{2} \mid Y_{1}=t}^{-}(0)-F_{X_{2} \mid X_{1}=t}^{-}(0)\right) d t \geq 0 .
\end{aligned}
$$

By using (iii) is immediately obtained that

$$
F_{Y_{2} \mid Y_{1}=t}^{-}(0)-F_{X_{2} \mid X_{1}=t}^{-}(0) \geq 0 \text { for all } t \in \mathbb{R} .
$$

Consequently,

$$
\int_{F_{1}^{-}(u)}^{+\infty} \bar{F}_{1}(t)\left(E\left(Y_{2} \mid Y_{1}=t\right)-E\left(X_{2} \mid X_{1}=t\right)\right) d t \geq 0, \forall u .
$$

Let $d W(t)=\bar{F}_{1}(t) \frac{f_{1}(t)}{\bar{F}_{1}(t)}\left(E\left(Y_{2} \mid Y_{1}=t\right)-E\left(X_{2} \mid X_{1}=t\right)\right) d t$ and $h(t)=t \cdot \frac{f_{1}(t)}{\bar{F}_{1}(t)}$. By assumption $(i i), h(t)$ is increasing, and, by inequality above, $\int_{s}^{+\infty} d W(t) \geq 0$ for all $s=F_{1}^{-}(u) \geq 0$.

Now, using Lemma 7.1 (a) in Barlow and Proschan (1975), it is obtained that

$\int_{0}^{+\infty} h(t) d W(t)=$

$\int_{0}^{+\infty} \bar{F}_{1}(t) \frac{f_{1}(t)}{\bar{F}_{1}(t)} t\left(E\left(Y_{2} \mid Y_{1}=t\right)-E\left(X_{2} \mid X_{1}=t\right)\right) d t=$

$\int_{0}^{+\infty} f_{1}(t)\left(E\left(t Y_{2} \mid Y_{1}=t\right)-E\left(t X_{2} \mid X_{1}=t\right)\right) d t \geq 0$.

Thus,

$$
E\left(Y_{1} Y_{2}\right) \geq E\left(X_{1} X_{2}\right)
$$

and by (i) and (ii) the result holds. Now, assume that $E\left(X_{2}\right) \neq E\left(Y_{2}\right)$. Note that $\mathbf{X} \leq_{e w} \mathbf{Y}$ if, and only if $\widetilde{\mathbf{X}} \leq_{e w}$ $\widetilde{\mathbf{Y}}$. Thus, in a similar manner, it is obtained that

$$
E\left(X_{1} \widetilde{X_{2}}\right) \leq E\left(Y_{1} \widetilde{Y_{2}}\right)
$$

and the result is immediately obtained.

The following corollary points out that, under appropriate assumption on aging for the first component, the excess wealth order can be considered as positive dependence order, since it implies the comparison between the covariances for vectors in the same Frechet class.

Corollary 1. Let $\mathbf{X}=\left(X_{1}, X_{2}\right)$ and $\mathbf{Y}=\left(Y_{1}, Y_{2}\right)$ be two bidimensional random vectors having the same marginal distributions. If $X_{1}$ has a IGFR distribution, then

$$
\mathbf{X} \leq_{\text {ew }} \mathbf{Y} \Rightarrow \operatorname{cov}\left(X_{1}, X_{2}\right) \leq \operatorname{cov}\left(Y_{1}, Y_{2}\right)
$$

\section{Acknowledgements}

The authors thank the anonymous referees and Prof. Alfonso Suárez-Lloréns for their valuable suggestions that helped to improve our work. 
Arias-Nicolás, J.P.; Fernández-Ponce, J.M.; Luque-Calvo, P. and Suárez-Lloréns, A. (2005), Multivariate dispersion order and the notion of copula applied to the multivariate $t$-distribution. Probability in the Engineering and Informational Sciences, 19, 363-375.

Arjas, E. and Lehtonen, T. (1978), Approximating many server queues by means of single server queues, Math. Oper. Res., 3, 205223.

Barlow, R.E. and Proshan, F. (1975), Statistical Theory of Reliability and Life Testing: Probability Models, (Hold, Rinehart and Winston.) NewYork

Bartoszewicz, J. (1995), Stochastic order relations and the total time on test transform, Statistics and Probability Letters, 22, 103-110.

Bartoszewicz, J. (1997), Dispersive functions and stochastic orders, Applicationes Mathematicae, 24, 429-444.

Belzunce, F; Candel, J. and Ruiz, J.M. (1998), Ordering and asymptotic properties of residual income distributions.Sankhya, Indian Journal of Statistics, 60, 331-348.

Belzunce, F., Ruiz, J.M. and Suárez-Lloréns, A. (2008), On multivariate dispersion ordering based on the standard construction. Statistics and Probability Letters, 78, 271-281.

Deshpandé, J.V. and Kochar, S.C. (1983), Dispersive ordering is the same as the tail-ordering, Advances in Applied Probability, 15, 686-687.

Fernández-Ponce, J.M., Infante-Macías, R. and Muñóz-Pérez, J (1996). Characterization of lifetime distributions based on a quantile dispersion measure, Computational Statistics \& Data Analysis, 21, $5,547-561$

Fernández-Ponce, J.M., Kochar, S.C. and Muñóz-Pérez, J. (1998) Partial orderings of distributions based on right-spread functions, Journal of Applied Probability, 35, 221-228.

Fernández-Ponce, J.M. and Suárez-Lloréns, A. (2003), A Multivariate Dispersion Ordering based on Quantiles More Widely Separated, Journal of Multivariate Analysis, 85, 40-53.

Hickey, R.J. (1986), Concepts of dispersion in distributions: a comparative note, Journal of Applied Probability, 23, 914-921.

Kochar, S.C. and Wiens, D.P. (1987), Partial orderings of life distributions with respect to their aging properties, Naval Research Logistics, 34, 823-829.

Kochar, S.C. (1996), Dispersive ordering of order statistics, Statistics and Probability Letters, 27, 271-274.

Kochar, S.C. and Carrière, K.C. (1997), Connections among various variability orderings, Statistics and Probability Letters, 35, 327333.

Kochar, S.C., Xiaohu Li and Shaked, M. (2001), The total time on test transform and the excess wealth stochastic orders of distributions, Advances in Applied Probabability, 34, (4), 826-845.

Lariviere, M.A. (2006), A note on probability distributions with increasing generalized failure rates. Operations Research, 54, 602604 .

Li, H.; Scarsini, M. and Shaked, M. (1996), Linkages: a tool for the construction of multivariate distribution with given nonoverlapping multivariate marginals. Journal of Multivariate Analysis, 56, 20-41.

Müller, A. and Scarsini, M. (2001), Stochastic comparison of random vectors with a common copula. Math. Oper. Res., 26, No. 4, 723740 .
Muñóz-Pérez, J. (1990), Dispersive ordering by the spread function, Statistics and Probability Letters, 10, 407-410.

O'Brien, G. (1975), The comparison method for stochastic processes, Ann. Prob., 3, 80-88.

Pellerey, F. and Shaked, M. (1997), Characterizations of the IFR and DFR aging notions by means of the dispersive order, Statistics and Probability Letters, 33, 389-393.

Rubinstein, R.Y., Samorodnitsky, G. and Shaked, M. (1985), Antithetic variates, multivariate dependence a simulation of complex systems. Management Science, 31, 66-77.

Rüschendorf, L. (1981), Stochastically ordered distributions and monotonicity of the OC-function of sequential probability ratio tests, Math. Operrationsforsch Statis., 12, 327-338.

Rüschendorf, L. (2004), Comparison of multivariate risks and positive dependence, Journal of Applied Probability, 41, 391-406.

Saunders, D.J. (1984), Dispersive orderings of distributions, Advances in Applied Probability, 16, 693-694.

Shaked, M. (1982), Dispersive Ordering of Distributions, Journal of Applied Probability, 19, 310-320.

Shaked, M. and Shanthikumar, J. G. (1994), Sthocastic Orders and Their Appications, (Academic Press, New York).

Shaked, M. and Shanthikumar, J.G. (1998), Two variability orders, Probability in the Engineering and Informational Sciences, 12, 1-23.

Stoyan, D. (1983), Comparison Methods for Queues and Other Stochastic Models, (Wiley, New York). 